\title{
Entanglement of Macroscopic Test Masses and the Standard Quantum Limit in Laser Interferometry
}

\author{
Helge Müller-Ebhardt, ${ }^{1}$ Henning Rehbein, ${ }^{1}$ Roman Schnabel, ${ }^{1}$ Karsten Danzmann, ${ }^{1}$ and Yanbei Chen ${ }^{2}$ \\ ${ }^{1}$ Max-Planck-Institut für Gravitationsphysik (Albert-Einstein-Institut), Institut für Gravitationsphysik, Leibniz Universität Hannover, \\ Callinstr. 38, 30167 Hannover, Germany \\ ${ }^{2}$ Max-Planck-Institut für Gravitationsphysik (Albert-Einstein-Institut), Am Mühlenberg 1, 14476 Potsdam, Germany
}

(Received 27 February 2007; published 7 January 2008)

\begin{abstract}
We show that the generation of entanglement of two heavily macroscopic mirrors is feasible with state of the art techniques of high-precision laser interferometry. The basis of such a demonstration would be a Michelson interferometer with suspended mirrors and simultaneous homodyne detections at both interferometer output ports. We present the connection between the generation of entanglement and the standard quantum limit (SQL) for a free mass. The SQL is a well-known reference limit in operating interferometers for gravitational-wave detection and provides a measure of when macroscopic entanglement can be observed in the presence of realistic decoherence processes.
\end{abstract}

DOI: 10.1103/PhysRevLett.100.013601

PACS numbers: 42.50.Xa, 03.65.Ta, 03.67.Mn, 42.50.Lc

The continuous quantum measurement of macroscopic objects was first investigated in the context of gravitational-wave (GW) detection [1]. In laser interferometer GW detectors, incoming GWs induce very weak, yet highly classical, tidal forces on mirror-endowed test masses. These are approximately free masses since they are hung from seismic isolation stacks as pendulums with eigenfrequency $(\sim 1 \mathrm{~Hz})$ much below detection band $(10 \mathrm{~Hz} \lesssim \Omega /(2 \pi) \lesssim 10 \mathrm{kHz})$. The interferometer measures the change in the test-mass mirror's relative positions [2]. The position observable, however, does not have commuting Heisenberg operators at different times, resulting in a standard quantum limit (SQL) [1]

$$
S_{\mathrm{SQL}} \equiv 2 \hbar /\left(m \Omega^{2}\right) \text {, }
$$

where $m$ is the reduced mirror mass. After a rather controversial debate it was eventually realized that the SQL can in principle be surpassed [3] if (i) measurement of the external force is made through monitoring of a quantum nondemolition observable [4], (ii) the quantum mechanics of the measuring device is taken into account, and appropriate quantum correlations are used, e.g., with backaction evasion techniques [5]. To reach the sensitivity required for GW astronomy, future GW detectors as well as near term prototypes will be designed to reach and even surpass the SQL [6-10] using either of the approaches mentioned. At the same time it is necessary to reduce all technical noise in order to reach this goal. These efforts are made with devices having test masses and arms ranging from gramscale and meter-long [10], kilogram-scale and $100 \mathrm{~m}$ [9] up to $40 \mathrm{~kg}$ and $4 \mathrm{~km} \mathrm{[6-8].}$

In this Letter, we show that a Michelson interferometer at its SQL produces entanglement between the conditional states of its otherwise free test-mass mirrors, if both common- and differential-mode test-mass motion are measured, but with different quantum-measurement processes-a direct mechanical analogy to the EinsteinPodolsky-Rosen gedanken experiment [11]. Contrary to former proposals [12], our work is aimed directly at experimental efforts in the GW community towards SQL sensitivity, and therefore may enable the first observation of entanglement between truly macroscopic objects. In technical terms, we introduce Wiener filtering [13], which, compared with the more conventional approach involving stochastic master equation (SME) [14], allows us to determine the conditional state much more directly from the interferometer's noise budget.

Configuration. - We consider an equal-arm Michelson interferometer as in Fig. 1, with laser (carrier) light injected from left. The two beams split at the beam splitter (BS) are reflected by identical mirrors in the north $(\mathrm{N})$ and east (E) arms, before being recombined at the BS. The south port is kept dark at the zero point, with all light reflected to the bright port. A power-recycling (PR) mirror is positioned such that it forms a resonant, but relatively low finesse, cavity for the carrier light with the test-mass

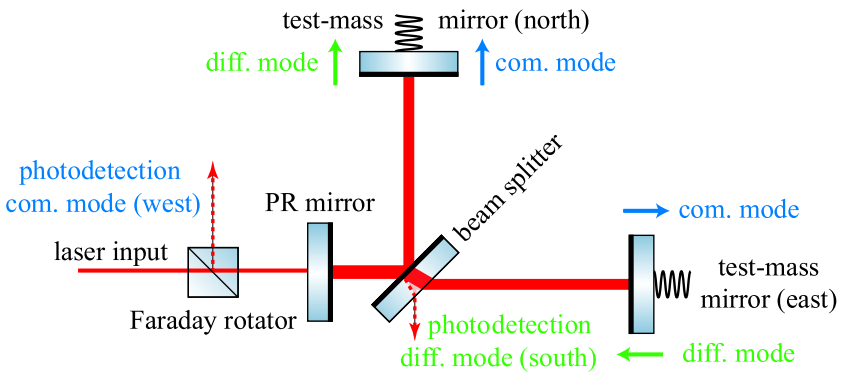

FIG. 1 (color online). Schematic plot of a power-recycled (PR) Michelson interferometer. Suspended test-mass mirrors are much lighter than other suspended optics. Differential motion of test-mass mirrors detected at dark (south) port and common motion at bright (west) port. A Faraday rotator might be used to access all of the back reflected light. 
mirrors. A differential (common) arm-length change induces phase-modulation fields that only emerge at the dark (bright) port. Correspondingly, fluctuating modulation fields that enter the interferometer from the dark (bright) port only interact with test-mass mirror displacements in the differential (common) mode. Homodyne detections are made at both, the dark and the bright port, each with a certain frequency-independent quadrature phase. In this way, we have two independent measurement processes in our interferometer, one for $\hat{x}^{d} \equiv\left(\hat{x}^{\mathrm{E}}-\hat{x}^{\mathrm{N}}\right)$ and the other for $\hat{x}^{c} \equiv\left(\hat{x}^{\mathrm{E}}+\hat{x}^{\mathrm{N}}\right)$. Defining $\hat{p}^{c, d} \equiv\left(\hat{p}^{\mathrm{E}} \pm \hat{p}^{\mathrm{N}}\right) / 2$, we have $\left[\hat{x}^{c, d}, \hat{p}^{c, d}\right]=i \hbar$. As it is well known, homodyne detections of out-going modulation fields collapse the quantum state of the corresponding mode of test-mass mirror motion. In absence of classical noise, each mode will reach a stochastic Gaussian pure state, with first-order moments $\langle\hat{x}\rangle$ and $\langle\hat{p}\rangle$ undergoing random walk, and second-order moments $V_{x x}, V_{x p}$ and $V_{p p}$ remaining constant and minimum-Heisenberg-limited, as required by purity. At any instant, values of $\langle\hat{x}(t)\rangle$ and $\langle\hat{p}(t)\rangle$ are determined by measurement results in the past. In this sense, this Gaussian state is called a posterior state [15]. As long as the measurement processes for the common and differential modes are different in terms of, e.g., signal storage time and/or homodyne phase, the wave functions $\psi^{c}$ and $\psi^{d}$ will be different, and the joint wave function $\Psi\left(x^{\mathrm{E}}, x^{\mathrm{N}}\right)=\psi^{c}\left[\left(x^{\mathrm{E}}+x^{\mathrm{N}}\right) / 2\right] \psi^{d}\left[\left(x^{\mathrm{E}}-x^{\mathrm{N}}\right) / 2\right]$ must be nonseparable. However, in presence of classical noise entanglement becomes less significant or even disappears. Note that this is analogous to creating entanglement by overlapping two differently squeezed beams on a BS [16].

Wiener filtering. - In the following we obtain the conditional quantum state using Wiener filtering [13], which applies to stable linear systems with Gaussian noise. More details will be given in Ref. [17]. Let $\hat{y}(t)$ be the Heisenberg operator of the out-going field quadrature being measured, and $\hat{x}(t)$ be the Heisenberg operator of the test-mass position. If we want to estimate $\hat{x}$ using the output $\hat{y}$, the optimal filter $K_{x}(t)$ is determined by the condition that the quantity

$$
\hat{R}_{x}(t) \equiv \hat{x}(t)-\int_{-\infty}^{t} d t^{\prime} K_{x}\left(t-t^{\prime}\right) \hat{y}\left(t^{\prime}\right)
$$

must be uncorrelated with the past output, i.e., $\left\langle\hat{R}_{x}(t) \hat{y}\left(t^{\prime}\right)+\hat{y}\left(t^{\prime}\right) \hat{R}_{x}(t)\right\rangle=0$ for all $t^{\prime}<t$. This leads to a Wiener-Hopf equation, whose solution can be given in terms of the single-sided (cross-) spectral densities of $\hat{x}$ and $\hat{y}: S_{x y}(\Omega), S_{x}(\Omega)$ and $S_{y}(\Omega) \equiv s_{y}(\Omega) s_{y}^{*}(\Omega)$ with $s_{y}(\Omega)$ and its inverse being analytic in the upper-half complex plane. Then $K_{x}(\Omega)=\left[S_{x y} / s_{y}^{*}\right]_{+} / s_{y}$. Note that $[\ldots]_{+}$ stands for taking the component of a function whose inverse Fourier transform has support only in positive times. The first- and second-order moments of the posterior state are then given by

$$
\langle\hat{x}(t)\rangle=\int_{-\infty}^{t} d t^{\prime} K_{x}\left(t-t^{\prime}\right) y\left(t^{\prime}\right)
$$

$$
V_{x x}=\int_{0}^{\infty} \frac{d \Omega}{2 \pi}\left(S_{x}-\left[S_{x y} / s_{y}^{*}\right]_{+}\left[S_{x y} / s_{y}^{*}\right]_{+}^{*}\right),
$$

respectively, where $y(t)$ is the measurement data of $\hat{y}(t)$. The same approach applies to the momentum operator $\hat{p}$ (replace every $x$ by $p$ in Eqs. (2)-(4)), and we also find

$$
V_{x p}=\int_{0}^{\infty} \frac{d \Omega}{2 \pi} \mathfrak{R}\left\{S_{x p}-\left[S_{x y} / s_{y}^{*}\right]_{+}\left[S_{p y} / s_{y}^{*}\right]_{+}^{*}\right\} .
$$

Such an essentially classical filtering is justified because $\left[\hat{y}(t), \hat{y}\left(t^{\prime}\right)\right]=0$ (satisfied by all out-going field quadratures) and $\left[\hat{x}(t), \hat{y}\left(t^{\prime}\right)\right]=\left[\hat{p}(t), \hat{y}\left(t^{\prime}\right)\right]=0$ for $t>t^{\prime}$ (due to causality, i.e., the out-going field does not influence any future test-mass observable). Furthermore, it can be shown that the conditional moments do not change when different linear feedback systems are applied-as long as at time $t$ the control force only depends on $\left\{\hat{y}\left(t^{\prime}\right): t^{\prime}<t\right\}$.

Posterior state of a single mode and squeezing. - Both the common and the differential-mode quantummeasurement process, can each be described by a set of two frequency-domain Heisenberg equations,

$$
\begin{aligned}
& \hat{y}(\Omega)=\sin \phi \hat{a}_{1}+\cos \phi\left[\hat{a}_{2}+\alpha / \hbar\left(\hat{x}(\Omega)+\hat{\xi}_{x}\right)\right], \\
& \hat{x}(\Omega)=-\left(\alpha \hat{a}_{1}+\hat{\xi}_{F}\right) /\left[m\left(\Omega^{2}+i \gamma_{m} \Omega-\omega_{m}^{2}\right)\right],
\end{aligned}
$$

with $\omega_{m}$ the pendulum angular frequency and $\gamma_{m}$ the pendulum damping rate. Equation (6) shows that $\hat{y}$ depends on the in-going amplitude or phase quadratures $\hat{a}_{1,2}$, the motion of the mirror center of mass, and sensing noise $\hat{\xi}_{x}$. The latter may be due to optical losses, or thermal fluctuations of the mirror's shape, i.e., internal thermal noise, which makes the mirror surface move with respect to its center of mass. Equation (7) describes the motion of the test-mass center of mass under radiation-pressure noise $\left(\alpha \hat{a}_{1}\right)$, as well as a classical force noise $\hat{\xi}_{F}$, acting directly on the center of mass of each test-mass mirror, e.g., due to seismic noise or suspension thermal noise. The quantity $\alpha$ indicates the measurement strength, given by $\alpha_{d}=$ $\sqrt{4 \hbar \omega_{0} P} / c$ for the differential mode and $\alpha_{c}=$ $2 / \tau \sqrt{4 \hbar \omega_{0} P} / c$ for the common mode, where $P$ is the circulating power in the arms and $\tau$ the PR-mirror transmissivity. The angle $\phi$ denotes the quadrature angle of $\hat{y}$. In the following we will only consider phase-quadrature readout, i.e., $\phi=0$.

From Eqs. (6) and (7) and with $S_{a_{i} a_{j}}=\delta_{i j}$ (referring to vacuum input) as well as $S_{\xi_{F}}$ and $S_{\xi_{x}}$, determined from the interferometer's noise budget, we obtain the relevant spectral densities: $S_{x}=\left[\alpha^{2}+S_{\xi_{F}}\right] /\left\{m^{2}\left[\left(\Omega^{2}-\omega_{m}^{2}\right)^{2}+\right.\right.$ $\left.\left.\gamma_{m}^{2} \Omega^{2}\right]\right\}, S_{x y}=(\alpha / \hbar) S_{x}$ and $S_{y}=1+(\alpha / \hbar)^{2}\left[S_{x}+S_{\xi_{x}}\right]$, as well as $S_{p}=m^{2} \Omega^{4} S_{x}, S_{p y}=-i m \Omega S_{x y}$, and $S_{x p}=$ $i m \Omega S_{x}$. For simplicity, we assume $\hat{\xi}_{F}$ and $\hat{\xi}_{x}$ to have a white spectrum, $S_{\xi_{F}}=2 \hbar m \Omega_{F}^{2}$ and $S_{\xi_{x}}=2 \hbar /\left(m \Omega_{x}^{2}\right)$, even though our approach enables us to treat nonwhite spectra. The sensitivity of the interferometer is given by the SQL-normalized, position-referred noise spectral density in the detection band, i.e., $\Omega \gg \omega_{m}, \gamma_{m}$ 


$$
\frac{S_{\text {total }}}{S_{\mathrm{SQL}}}=\underbrace{\frac{1}{2}\left(\frac{\Omega^{2}}{\Omega_{\alpha}^{2}}+\frac{\Omega_{\alpha}^{2}}{\Omega^{2}}\right)}_{S_{\text {quant }} / S_{\mathrm{SQL}}}+\underbrace{\Omega_{F}^{2} / \Omega^{2}}_{S_{\text {force }} / S_{\mathrm{SQL}}}+\underbrace{\Omega^{2} / \Omega_{x}^{2}}_{S_{\text {sens }} / S_{\mathrm{SQL}}} .
$$

The quantum noise $S_{\text {quant }}$ is limited from below by $S_{\mathrm{SQL}}$ with equality reached at $\Omega_{\alpha} \equiv \alpha / \sqrt{m \hbar}$ which sets a time scale for the measurement process. The force and sensing noise $S_{\text {force }}$ and $S_{\text {sens }}$ intersect $S_{\mathrm{SQL}}$ at $\Omega_{F}$ and $\Omega_{x}$, respectively. For $\Omega_{x} / \Omega_{F}>2$, we have a nonzero frequency band (in between $\Omega_{F}$ and $\Omega_{x}$ ) in which the classical noise is completely below the SQL (cf. Figure 2). Solving the Wiener filtering problem, we obtain in the limit of $\gamma_{m}$, $\omega_{m} \ll \Omega_{\alpha}$

$$
\begin{gathered}
V_{x x}=\hbar /\left(\sqrt{2} m \Omega_{\alpha}\right)\left[\left(1+2 \zeta_{F}^{2}\right)\left(1+2 \zeta_{x}^{2}\right)^{3}\right]^{1 / 4}, \\
V_{p p}=\hbar m \Omega_{\alpha} / \sqrt{2}\left[\left(1+2 \zeta_{F}^{2}\right)^{3}\left(1+2 \zeta_{x}^{2}\right)\right]^{1 / 4}, \\
V_{x p}=\hbar / 2\left[\left(1+2 \zeta_{F}^{2}\right)\left(1+2 \zeta_{x}^{2}\right)\right]^{1 / 2},
\end{gathered}
$$

for the posterior state, where we have defined $\zeta_{F} \equiv$ $\Omega_{F} / \Omega_{\alpha}$ and $\zeta_{x} \equiv \Omega_{\alpha} / \Omega_{x}$. At the bright port the in-going modulation fields at frequencies $\Omega$ are usually not in vacuum states, i.e. $S_{a_{1} a_{1}}, S_{a_{2} a_{2}}>1$ due to technical laser noise, which adds for the common mode $\left(S_{a_{1} a_{1}}-1\right) / 2$ and $\left(S_{a_{2} a_{2}}-1\right) / 2$ to $\zeta_{F}^{2}$ and $\zeta_{x}^{2}$, respectively. Note that Eqs. (9)-(11) are consistent with results obtained from SME [18], although with a more complicated noise budget, solving the Wiener Filtering problem will be substantially easier.

A measure of the quantum nature of a state is the quantity $U=V_{x x} V_{p p}-V_{x p}^{2}$, with $U \geq \hbar^{2} / 4$, the Heisenberg uncertainty principle. According to Eqs. (9)-(11),

$$
U /\left(\hbar^{2} / 4\right)=\left(1+2 \zeta_{F}^{2}\right)\left(1+2 \zeta_{x}^{2}\right) \geq\left(1+2 \Omega_{F} / \Omega_{x}\right)^{2},
$$

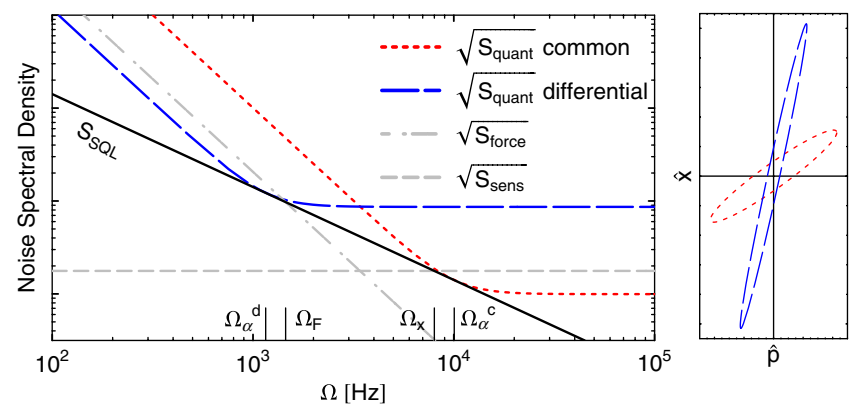

FIG. 2 (color online). Noise spectral densities (left panel) in arbitrary units with $\Omega_{F}=2 \pi 230 \mathrm{~Hz}$ and $\Omega_{x}=2 \pi 1270 \mathrm{~Hz}$, and optimized $\Omega_{\alpha}^{c}=2 \pi 1600 \mathrm{~Hz}, \Omega_{\alpha}^{d}=2 \pi 184 \mathrm{~Hz}$. Test-mass entanglement of $E_{\mathcal{N}}=0.35$. Squeezing ellipses of position and momentum operators (right panel) with respect to common (red) and differential (blue) mode. Second-order moments are normalized to the ground state of harmonic oscillator at frequency $\left(\Omega_{\alpha}^{c}+\Omega_{\alpha}^{d}\right) / 2$. with equality achieved when $\Omega_{\alpha}=\sqrt{\Omega_{x} \Omega_{F}}$. We see that as long as $\zeta_{F}$ and $\zeta_{x}$ are not too big, the posterior state is highly squeezed in position, and highly antisqueezed in momentum with respect to the ground state of the pendulum (recall that $\Omega_{\alpha} \gg \omega_{m}$ ). Increasing (decreasing) $\Omega_{\alpha}$, e.g., by varying the circulating power or the PR-mirror transmissivity, results in more (less) squeezing in position, and more (less) antisqueezing in momentum - causing the squeezing ellipse to rotate, as shown in Fig. 2. The posterior state can also be modified when a different outputquadrature phase $\phi$ is used.

Entanglement. - After obtaining the individual common- and differential-mode posterior states, we assemble the posterior state of the entire system. The combined $(4 \times$ 4) covariance among $\left(x^{\mathrm{E}}, p^{\mathrm{E}}, x^{\mathrm{N}}, p^{\mathrm{N}}\right)$ reads $V=$ $\left[\left(V_{\mathrm{EE}}, V_{\mathrm{EN}}\right),\left(V_{\mathrm{NE}}, V_{\mathrm{NN}}\right)\right]$ with

$$
\begin{aligned}
& V_{\mathrm{NN}}=V_{\mathrm{EE}}=\left(\begin{array}{cc}
\left(V_{x x}^{c}+V_{x x}^{d}\right) / 4 & \left(V_{x p}^{c}+V_{x p}^{d}\right) / 2 \\
\left(V_{x p}^{c}+V_{x p}^{d}\right) / 2 & V_{p p}^{c}+V_{p p}^{d}
\end{array}\right), \\
& V_{\mathrm{EN}}=V_{\mathrm{NE}}=\left(\begin{array}{cc}
\left(V_{x x}^{c}-V_{x x}^{d}\right) / 4 & \left(V_{x p}^{c}-V_{x p}^{d}\right) / 2 \\
\left(V_{x p}^{c}-V_{x p}^{d}\right) / 2 & V_{p p}^{c}-V_{p p}^{d}
\end{array}\right) .
\end{aligned}
$$

A computable measure of entanglement, the logarithmic negativity, for an arbitrary bipartite system was introduced in [19]. For our state

$$
E_{\mathcal{N}}=\max \left[0,-\log _{2} 2 \sigma^{-} / \hbar\right],
$$

where $\sigma^{-}=\sqrt{\left(\Sigma-\sqrt{\left.\Sigma^{2}-\overline{4} \overline{\operatorname{det}} \bar{V}\right) / 2}\right.}$ and $\Sigma=\operatorname{det} V_{\mathrm{NN}}+$ $\operatorname{det} V_{\mathrm{EE}}-2 \operatorname{det} V_{\mathrm{NE}}$. If we insert Eqs. (9)-(11) into Eq. (15), only three parameter ratios remain: $\Omega_{\alpha}^{c, d} / \Omega_{F}$ for common and differential mode, respectively, and $\Omega_{x} / \Omega_{F}$. The latter turns out to be the crucial factor for the existence of test-mass entanglement. The parameters in Fig. 2 result in $E_{\mathcal{N}}=0.35$. Recall that there exists a frequency window with sub-SQL classical noise iff $\Omega_{x} / \Omega_{F}>2$. However, the existence of entanglement sets a slightly higher threshold value for this frequency ratio. In Fig. 3, we plot the maximum achievable $E_{\mathcal{N}}$ (varying $\Omega_{\alpha}^{c}$ and $\Omega_{\alpha}^{d}$ ) as a function of $\Omega_{x} / \Omega_{F}$ for different strengths of

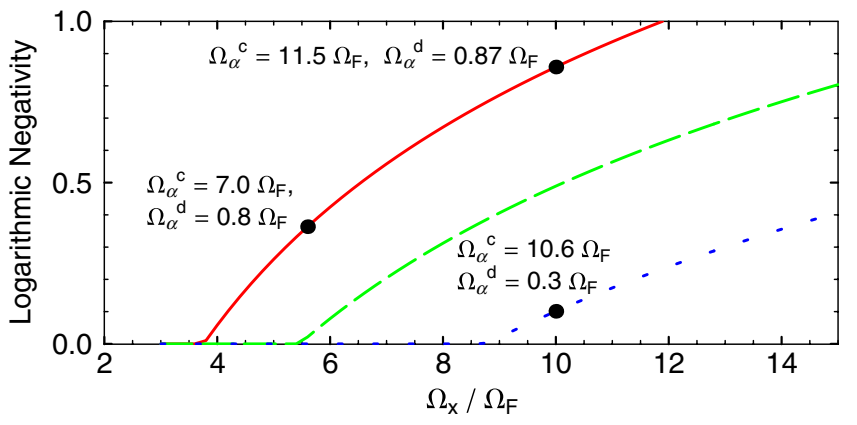

FIG. 3 (color online). Logarithmic negativity versus $\Omega_{x} / \Omega_{F}$, maximized with respect to $\Omega_{\alpha}^{c}$ and $\Omega_{\alpha}^{d}$ (example values marked) for $0 \mathrm{~dB}$ (solid line), $5 \mathrm{~dB}$ (dashed line) and $10 \mathrm{~dB}$ (dotted line) technical laser noise, in both, amplitude and phase quadrature. 
technical laser noise. Remarkably, even for a value of $10 \mathrm{~dB}$ above vacuum noise, the generation of entanglement is still possible.

Feasibility of achieving sub-SQL classical noise.Current experiments [6-10] aim to reach or even surpass the SQL by reducing the classical noise around $\Omega_{\alpha}$ which is in fact not the optimum strategy for strong test-mass entanglement (cf. Fig. 2). Furthermore, these experiments only consider a single readout. Nevertheless, they provide valuable information whether sub-SQL classical noise is achievable at all. Regarding the force noise, it is feasible to suppress the dominant suspension thermal noise below the SQL in the detection band even at room temperature. This can be realized by a low pendulum frequency with a high quality factor $\omega_{m} / \gamma_{m}$. In Advanced LIGO [8], we expect $\Omega_{F} /(2 \pi) \sim 40 \mathrm{~Hz}$ and $\Omega_{\alpha} /(2 \pi)$ marginally higher. In labscale experiments $\Omega_{F} /(2 \pi)=400 \mathrm{~Hz}$ and $\Omega_{\alpha} /(2 \pi)$ of several $\mathrm{kHz}$ have already been realized [10]. Regarding the sensing noise, optical losses need to be discussed [7]. In our setup the main contribution arises from photodetection loss because no high-finesse cavities are involved. Assuming a total optical loss in power of $\epsilon=2 \%$, this on its own would imply $\Omega_{x}=14 \Omega_{\alpha}$, which is more than sufficient. More challenging is mirror internal thermal noise. In Advanced LIGO, we expect $\Omega_{x}$ to roughly coincide with $\Omega_{\alpha}$. However, cooled test masses will allow to suppress this noise and increase $\Omega_{x}$. In the CLIO interferometer currently being commissioned, the theoretical thermal-noise budget suggests $\Omega_{x} / \Omega_{F} \sim 10$ at a temperature of $20 \mathrm{~K}$ [9]. In the configuration outlined in Ref. [10], we have $\Omega_{x} /(2 \pi)=50 \mathrm{~Hz}$, which is a factor of 8 below $\Omega_{F}$. With a new test-mass with lower mechanical coating loss, we expect $\Omega_{x}$ to increase by a factor of $\sim 1.5$. Increasing the beam spot size by factor of 3 , will provide an additional factor of $3^{2}$ [10], allowing $\Omega_{x} / \Omega_{F} \sim 1.7$, even at room temperature. Other strategies are currently being investigated to further suppress the internal thermal noise [20]. Laser amplitude noise can be suppressed sufficiently with current technology [21]. Regarding laser phase noise, a level of $10 \mathrm{~dB}$ above vacuum noise - for an input laser power of, e.g., $1 \mathrm{~W}$ at a measurement frequency of $1 \mathrm{kHz}$-corresponds to a frequency noise of about $10^{-5} \mathrm{~Hz} / \sqrt{\mathrm{Hz}}$. Reference [22] suggests that this stability has already been reached for the injected carrier light of current GW detectors.

Conclusion. - In this Letter, we obtain a general and quantitative condition for the possibility of generating macroscopic entanglement between two test-mass mirrors of a Michelson interferometer-in terms of the interferometer's classical noise. We have found that these classical noise sources are required to surpass the SQL only moderately, well within the reach for experiments to be carried out in the GW community within the next decade.
We thank the AEI-Caltech-MIT MQM group for interesting discussions, in particular, T. Corbitt, C. Li, N. Mavalvala, Y. Mino, K. Somiya, K. S. Thorne, S. Waldman, and C. Wipf. We thank B. Willke and S. Gossler for advice on laser noise, N. Mavalvala and C. Wipf for comments that helped us to clarify the message of this Letter, as well as Y. K. Lau, W. Kells, H. J. Kimble, and $\mathrm{H}$. Mabuchi for comments on our manuscript. This work has been supported by the Alexander von Humboldt Foundation's Sofja Kovalevskaja programme and by the DFG through the EGC programme and the SFB No. 407.

[1] C. M. Caves et al., Rev. Mod. Phys. 52, 341 (1980); V. B. Braginsky and F. Y. Khalili, Quantum Measurement (Cambridge University Press, Cambridge, England, 1999); V. B. Braginsky, Sov. Phys. JETP 26, 831 (1968).

[2] A. Abramovici et al., Science 256, 325 (1992).

[3] W. G. Unruh, Quantum Optics, Experimental Gravitation, and Measurement Theory (Plenum, New York, 1982), p. 647; C. M. Caves, Phys. Rev. Lett. 54, 2465 (1985); M. T. Jaekel and S. Reynaud, Europhys. Lett. 13, 301 (1990).

[4] V. B. Braginsky and F. Y. Khalili, Rev. Mod. Phys. 68, 1 (1996).

[5] K. S. Thorne et al., Phys. Rev. Lett. 40, 667 (1978).

[6] P. Fritschel, Proceedings of the SPIE (SPIE, Bellingham, WA, 2003), Vol. 4856, p. 282.

[7] H. J. Kimble et al., Phys. Rev. D 65, 022002 (2001).

[8] Advanced LIGO Reference Design, Report No. LIGO060056-07-M, 2006.

[9] S. Miyoki et al., Classical Quantum Gravity 21, S1173 (2004).

[10] T. Corbitt et al., Phys. Rev. A 73, 023801 (2006); Phys. Rev. Lett. 98, 150802 (2007).

[11] A. Einstein et al., Phys. Rev. 47, 777 (1935).

[12] S. Mancini et al., Phys. Rev. Lett. 88, 120401 (2002); J. Zhang et al., Phys. Rev. A 68, 013808 (2003); M. Pinard et al., Europhys. Lett. 72, 747 (2005); S. Pirandola et al., Phys. Rev. Lett. 97, 150403 ( 2006).

[13] N. Wiener, The Extrapolation, Interpolation, and Smoothing of Stationary Time Series (MIT, Cambridge, MA, 1949).

[14] G. J. Milburn, Quantum Semiclass. Opt. 8, 269 (1996).

[15] A. Barchielli, Int. J. Theor. Phys. 32, 2221 (1993).

[16] A. Furusawa et al., Science 282, 706 (1998); W. P. Bowen et al., Phys. Rev. Lett. 90, 043601 (2003).

[17] Y. Chen et al. (to be published).

[18] A. Hopkins et al., Phys. Rev. B 68, 235328 (2003).

[19] G. Vidal and R.F. Werner, Phys. Rev. A 65, 032314 (2002).

[20] A. Bunkowski et al., Classical Quantum Gravity 23, 7297 (2006); R. O'Shaughnessy, Classical Quantum Gravity 23, 7627 (2006); M. Bondarescu and K. S. Thorne, Phys. Rev. D 74, 082003 (2006).

[21] F. Seifert et al., Opt. Lett. 31, 2000 (2006).

[22] J. B. Camp et al., J. Opt. Soc. Am. A 17, 120 (2000). 